\title{
La transplantation de foie chez l'en- fant: un investissement pour la vie
}

\author{
Barbara Wildhaber ${ }^{a}$, Valérie McLin ${ }^{b}$, Dominique Belli ${ }^{c}$ \\ a Prof., Service de chirurgie pédiatrique; ${ }^{b}$ Prof., Unité de gastroentérologie pédiatrique, Service des spécialités pédiatriques; ${ }^{c}$ Prof., Service de pédiatrie; \\ Centre Suisse des Maladies du Foie de l'Enfant, Département de l'enfant et de l'adolescent, Hôpitaux Universitaires de Genève
}

\section{La transplantation hépatique chez l'enfant est un traitement médico-chirurgical hautement complexe et extrêmement demandeur en ressources humaines et médico-techniques. Elle constitue aujourd'hui le traitement de choix des maladies terminales du foie de l'enfant. Elle permet à des jeunes patients dont le pronostic vital est menacé de devenir un jour des citoyens actifs, des parents. Miser sur leur santé est donc un investissement pour notre société!}

La première transplantation de foie est réalisée à Denver, Colorado, en 1963, par le Professeur Thomas Starzl sur un enfant de trois ans porteur d'une atrésie des voies biliaires (une obstruction des voies biliaires d'origine inconnue, survenant en période périnatale) qui décédera d'hémorragie durant l'opération. La même année, quatre autres interventions sont effectuées chez des adultes qui se révéleront également infructueuses. Il faudra attendre le 17 mars 1971 pour voir la première greffe de foie réussie chez un enfant de 17 mois à Louvain, Belgique, par le Professeur Jean-Bernard Otte. Les taux de survie vont rester très modestes jusqu'à l'arrivée de la ciclosporine dans les années 1980. Ce puissant traitement immunosuppresseur va permettre de développer la transplantation d'organes solides. Par la suite, les techniques chirurgicales vont s'améliorer avec l'apparition des foies réduits et partagés, ainsi qu'avec l'alternative de donneurs vivants.

La survie à long terme des enfants transplantés du foie est aujourd'hui à 95\%.

C'est le $1^{\text {er }}$ juillet 1989 que la première greffe de foie chez l'enfant est réalisée en Suisse aux Hôpitaux Universitaires de Genève (HUG), initialement dans le cadre d'un programme de greffe de foie adulte-enfant. Depuis, ce programme s'est spécialisé à l'enfant, en restant en étroite collaboration avec l'équipe adulte, et en élargissant la coopération au niveau national avec une équipe médico-chirurgicale et multidisciplinaire. En Suisse, les transplantations hépatiques pédiatriques sont donc réalisées dans un seul centre, le Centre Suisse des Maladies du Foie de l'Enfant des HUG. A ce jour, plus de 170 transplantations pédiatriques ont été effectuées et actuellement 10 greffes en moyenne sont réalisées chaque année à Genève, ce qui répond aux besoins de la population helvétique.

Alors qu'il y a 20 ans, la perspective d'une greffe hépatique évoquait avant tout la question de la survie du patient et était synonyme de nombreuses complications, aujourd'hui, nous pouvons rassurer les familles, car même si la prise en charge est complexe, l'issue est le plus souvent excellente en termes de qualité de vie et de longévité.

\section{L'évolution remarquable de la greffe de foie chez l'enfant}

En Suisse, les indications à la greffe de foie sont comparables à celles des centres de transplantation dans le reste du monde, dans la moitié des cas étant l'atrésie des voies biliaires, une affection congénitale de l'arbre biliaire. Autrefois, le taux de survie de ces enfants était de $30-55 \%$ après 5 ans, de $30 \%$ après 10 ans et de $20 \%$ après 20 ans, alors qu'avec la greffe de foie il approche aujourd'hui les $100 \%$. Les enfants qui ont besoin d'une transplantation de foie sont souvent jeunes et petits, avec 50\% des enfants pesant moins de $10 \mathrm{~kg}$ lors de la greffe, deux tiers moins de $15 \mathrm{~kg}$. Les premières transplantations hépatiques pédiatriques duraient environ 12 heures, mais aujourd'hui, grâce à l'expérience de l'équipe chirurgicale et anesthésique, la durée s'est raccourcie à la moitié. La durée d'hospitalisation est également en nette diminution, et se situe de nos jours entre 2 et 4 semaines, alors que dans les années 90 les enfants restaient souvent hospitalisés pendant une très longue période qui était pesante et coûteuse pour les petits patients et leurs familles. La mortalité et la 
morbidité de la transplantation hépatique chez l'enfant sont dues à ses aspects chirurgicaux (saignement, thromboses vasculaires, complications biliaires, etc.) et médicaux (non-fonction primaire, infection, etc.). Ces derniers se rencontrent le plus souvent pendant la première année après la greffe et requièrent un savoirfaire très spécialisé. La survie globale des enfants après transplantation hépatique dans la série suisse de Genève a vu une évolution saisissante: pendant les 5 premières années du programme, nous devions faire face à 13\% de mortalité, aujourd'hui, entre 2011 et 2016, elle est à $5 \%$. La survie du greffon a vu une évolution encore meilleure et est montée de $72 \%$ entre 1989 et 1994 à 89\% entre 2011 et 2016.

\section{Le but ultime: développement et qualité de vie}

Au début, le but de la greffe de foie était de permettre aux enfants malades de survivre. Aujourd'hui, au-delà de l'aspect de survie, nous nous focalisons surtout sur une quasi-normalisation de la qualité de vie des patients, et tentons de ne pas remplacer une maladie chronique par une autre. Les patients peuvent et doivent pouvoir vivre une vie comme un enfant sain,

\section{Normalement, les enfants peuvent retourner} à l'école quelques semaines après la greffe, faire du sport, dans la majorité des cas apprendre le métier de leur choix et avoir des enfants.

retourner à l'école quelques semaines après la greffe, avoir des contrôles ambulatoires sans hospitalisations qui sont chronophages pour l'enfant et ses parents, fréquenter les colonies de vacances, dans la majorité des cas apprendre leur métier de choix, se permettre un planning familial. C'est pour cela que les équipes qui suivent ces enfants doivent être multidisciplinaires, spécialisées et compétentes dans toutes ces spécificités, afin d'offrir aux enfants et aux adolescents une survie optimale avec une qualité de vie excellente, même s'ils continuent de recevoir des traitements médicaux, mais peu contraignants, à long terme.
Sur le plan du développement psychomoteur et/ou intellectuel, les performances scolaires des patients greffés restent en moyenne légèrement inférieures à celles de la population générale, probablement en lien avec la maladie menant à la greffe. Néanmoins, le fonctionnement de ces patients à l'âge adulte est très satisfaisant tant au niveau professionnel que personnel. Le meilleur exemple de cette évolution tant favorable est la possibilité d'avoir des enfants.

En conclusion, la transplantation hépatique chez l'enfant est un exemple très illustratif de l'alliance entre la médecine hautement spécialisée et la multidisciplinarité pour non seulement augmenter la survie des patients, mais aussi assurer leur qualité de vie et leur avenir en tant que citoyens.

\section{Résumé}

La transplantation hépatique chez l'enfant est le traitement de choix pour les pathologies hépatiques chroniques et aiguës terminales. C'est un traitement médico-chirurgical hautement complexe et extrêmement demandeur en ressources humaines et médico-techniques qui a souvent été critiqué pour sa mortalité et sa morbidité. Or, la survie globale des enfants après transplantation hépatique a vu une évolution saisissante: pendant les 5 premières années du programme genevois, la mortalité était de 13\%, aujourd'hui elle est de $5 \%$, et la survie du greffon est montée de 72 à $89 \%$. Aujourd'hui, les patients jouissent d'une vie qui se rapproche de celle d'un enfant sain et leur fonctionnement à l'âge adulte est satisfaisant tant au niveau professionnel que personnel. Ainsi, la transplantation hépatique chez l'enfant est un très bon exemple où médecine hautement spécialisée et collaborations multidisciplinaires permettent non seulement d'augmenter la survie des patients, mais aussi d'assurer leur qualité de vie et leur avenir en tant que citoyens.

\section{Remerciements}

Les auteurs remercient Simona Korff, PhD, pour l'entretien du registre suisse des enfants transplantés du foie et le calcul des statistiques locales. 